\title{
Neuron-specific analysis of histone modifications with post-mortem brains
}

\author{
Kagari Koshi-Mano ${ }^{1}$, Tatsuo Mano ${ }^{1}$, Maho Morishima ${ }^{2}$, Shigeo Murayama ${ }^{2}$, Akira Tamaoka ${ }^{3}$, \\ Shoji Tsuji ${ }^{1}$, Tatsushi Toda ${ }^{1}$ \& Atsushi Iwata ${ }^{1 *}$
}

Histone modifications govern chromatin structures and regulate gene expression to orchestrate cellular functions in the central nervous system, where neuronal cells are postmitotic and developmentally inactive, the functional and age-dependent changes also accumulate in the epigenetic states. Because the brain is composed of several types of cells, such as the neurons, glial cells, and vascular cells, the analysis of histone modifications using bulk brain tissue might obscure alterations specific to neuronal cells. Furthermore, among the various epigenetic traits, analysis of the genome-wide distribution of DNA methylation in the bulk brain is predominantly a reflection of DNA methylation of the nonneuronal cells, which may be a potential caveat of previous studies on neurodegenerative diseases using bulk brains. In this study, we established a method of neuron-specific ChIP-seq assay, which allows for the analysis of genome-wide distribution of histone modifications specifically in the neuronal cells derived from post-mortem brains. We successfully enriched neuronal information with high reproducibility and high signal-to-noise ratio. Our method will further facilitate the understanding of neurodegeneration.

Histone modification is a part of the epigenome that includes covalent post-translational methylation, acetylation, or ubiquitylation of histone proteins. These modifications co-operate with other epigenetic factors ${ }^{1}$ such as DNA methylation or non-coding RNA, to alter chromatin structures, orchestrate gene expression, and regulate cellular functions, including cell division, growth, and differentiation during the developmental process ${ }^{2,3}$. In the central nervous system, where mature neuronal cells are postmitotic and developmentally inactive, histone modifications play a key role in memory formation and learning process contributing to neuronal plasticity ${ }^{4,5}$. Aging is also associated with chromatin remodeling, and a better understanding of the phenomenon could be leveraged to induce a variety of responses to restore youthful functionalities in old tissues ${ }^{6-8}$. Furthermore, in neurodegenerative conditions, such as Alzheimer's and Parkinson's disease, profound effects on histone modifications are thought to reflect the pathogenic neurodegenerative processes ${ }^{9,10}$. As a result, histone modifications that exist in mature neuronal cells have a complex structure, post hoc modifications corresponding to physiological and/or pathological process, layered on a priori modification specific to neuronal cells. Thus, genome-wide profiles of histone modifications specific to neuronal cells can facilitate the elucidation of physiological mechanisms of the brain related to learning and memory, and pathomechanisms, where various life-long factors converge to cause neurodegeneration.

When analyzing histone modifications in brain samples, we must consider the fact that the brain is composed of several types of cells, including neuronal cells that directly contribute to learning and memory, glial cells that support neuronal activities or provoke inflammation, and vascular cells that deliver oxygen and nutrition to the brain. Each type of cell has its own specific histone modification corresponding to its developmental process, and subsequently acquires alterations in the modifications based on its physiological and pathological condition. Therefore, histone modification of the bulk brain derived from the cerebral cortex is a mixture of that of neuronal and non-neuronal origins. Considering that neurons comprise approximately $40 \%{ }^{11-13}$ of all the cells in the cortex, bulk brain analysis is not representative of the neuronal epigenome. Thus, we hypothesized that the genome-wide profiles of histone modification in neuronal cells cannot be estimated by using bulk brain tissue,

${ }^{1}$ Department of Neurology, Graduate School of Medicine, The University of Tokyo, 7-3-1 Hongo, Bunkyo-ku, Tokyo, 113-8655, Japan. 2Department of Neuropathology, Tokyo Metropolitan Geriatric Hospital, 35-2 Sakaecho, Itabashi, Tokyo, 173-0015, Japan. ${ }^{3}$ Department of Neurology, University of Tsukuba, 1-1-1 Tennodai, Tsukuba, Ibaraki, 3058575, Japan. *email: iwata-tky@umin.ac.jp 
and this motivated us to develop a method for understanding the genome-wide profiles of histone modifications specific to neuronal cells.

Chromatin immunoprecipitation sequencing (ChIP-seq) is a method used to identify genome-wide profiles of histone modifications, where the genomic DNA that is wrapped around histone proteins is co-immunoprecipitated using a modification-specific anti-histone antibody to prepare libraries for next generation sequencing. For neuron-specific analysis, we applied fluorescence activated cell sorting (FACS)-based isolation of neuronal nuclei. Formerly, large number of cells was required for robust and reproducible ChIP-seq analysis and this used to be a major challenge for FACS isolation of neuronal nuclei where the number of the nuclei that could be isolated was limited. Especially, for studying neurodegenerative conditions where post-mortem brain samples are used and the amount of sample available for the assay is limited, the number of the nuclei required for the assay should ideally be low. The condition of the sample used in the assays is also critical for reproducibility because post-mortem brain samples are inevitably affected by the post-mortem time to autopsy and subsequent freeze-thaw processes. To overcome these issues, we optimized each step of the FACS and ChIP-seq that enabled multiple genome-wide histone modification analyses. Here, we demonstrate that neuron-specific histone modifications are completely different from non-neuron-specific, and bulk brain histone modifications, emphasizing the importance of neuronal isolation for post-mortem brain epigenome analysis.

\section{Results}

Optimization of crosslinking methods. The first step in the ChIP assay is the crosslinking of the nucleosome, which is composed of genomic DNA wrapped around histone proteins, and uniform reaction across the tissue is essential for reproducibility ${ }^{14}$. Generally, fixation in the early steps ensures optimum crosslinking. However, when using tissue sample, fixing brain tissue en block has a serious disadvantage in that the surface of the brain may be fixed more than its inside potentially leading to uneven ChIP-seq assay. Given that the separation of neuronal nuclei from non-neuronal ones using FACS is required to achieve specificity in neuronal ChIP-seq ${ }^{15}$, optimization of this step is crucial. Therefore, we tested two different time points for fixing the nucleosome complexes; (1) immediately after homogenization of the frozen brain or (2) after FACS. All the brains were obtained from the patients without any pathological conditions in the brain. When compared to the yield of genomic DNA extracted before DNA fragmentation, the yield was higher and more reproducible when the nuclei were fixed immediately after homogenization (Mean \pm SD: $26.2 \pm 8.4 \%$ vs $8.5 \pm 10.2 \%$ ) (Fig. 1a). We speculated that this was because before fixation the bare nuclear membrane can be easily fragmented during FACS. With this method, we obtained $47.4 \pm 19.3$ neuronal nuclei and $78.8 \pm 30.1$ non-neuronal nuclei from $100 \mathrm{mg}$ of the brain tissue (Fig. S1a). Separation of neuronal and non-neuronal nuclei confirmed by immunofluorescence staining and western blotting (Figs. $2 \mathrm{a}$ and S1b). On the completion of nuclear isolation, the neuronal or non-neuronal nuclei were subjected to sonication to fragment the genomic DNA into lengths of $150-250 \mathrm{bp}$ according to the standard sonication protocol, such that the genomic DNA was fragmented into single nucleosome units (Fig. S2).

Optimization of ChIP assay. We then optimized the amount of antibodies used in the immunoprecipitation of two representative histone modifications, $\mathrm{H} 3 \mathrm{~K} 4 \mathrm{me} 3$ and $\mathrm{H} 3 \mathrm{~K} 27 \mathrm{ac}$, that are positively correlated with gene expressions ${ }^{16}$. In general, the amount of DNA fragments captured by antibodies depends on the amount of antibodies used for the immunoprecipitation, however, excessive amounts of antibodies could potentially result in non-specific binding and increase undesirable signal in regions without target modifications. On the other hand, a low yield of DNA fragments requires more PCR cycles resulting in reduced library complexity and skewing of the library. Thus, optimization of the amount of antibody is crucial to obtain a good quality library with high sensitivity and specificity. To validate the yield and specificity, we performed qPCR with the immunoprecipitated DNA fragments. To assess the specificity of our neuronal and non-neuronal ChIP, we chose three genomic regions for qPCR as below, where are supposed to be enriched with the histone modifications analyzed in this study according to their expressions. ChIP reaction was performed using $2 \times 10^{6}$ nuclei in the reaction volume of $1 \mathrm{~mL}$. We measured the fold enrichment of TSS regions of GAPDH (endogenous positive control), GRIN2B (neuron-specific marker) and $H B B$ (negative control that is not expressed in the central nervous system) $)^{17}$. Along with the increasing amounts of antibodies, the fold enrichment of GAPDH, which is the universally expressed gene in all tissues, was enhanced (Fig. 1b), however, the signal from the negative control gene $H B B$, that is not expressed in the central nervous system, also increased thus lowering the signal to noise ratio (Fig. 1c). The TSS region of GRIN2B was enriched only in neuronal samples, which was consistent with specific expression of GRIN2B in neuronal cells. Based on these data, we determined that the optimal amount of antibody per assay is $0.1 \mu \mathrm{g}$ for anti-H3K4me3 and $5 \mu \mathrm{g}$ for anti-H3K27ac antibody. To validate the above-established ChIP method, we performed ChIP using more brain samples and qPCR to measure fold enrichment of the above regions, demonstrating the robustness of our method. Furthermore, we performed qPCR with the ChIP samples in other genomic regions including SYN3 and BDNF, neuron-specific genes, and ERMN and OLIG2, non-neuronal genes. As expected, SYN3 and BDNF were enriched only in neuronal ChIP samples, and ERMN and OLIG2 in non-neuronal ChIP samples. Taken together, we could confirm neuronal-enrichment in our neuron-specific ChIP in the local genomic regions, thus, we moved on to the genome wide validation of our method.

The DNA fragments were subjected to library preparation. To check the quality of the library, the fold enrichment of the three genes, GAPDH, GRIN2B, and $H B B$, was validated just before sequencing, which demonstrated that the library preparation process did not change the enrichment patterns (Fig. S3). Consistent with the result of qPCR in Fig. 3, the distributions of the mapped reads of the genes analyzed showed distinctive patterns according to neuronal or non-neuronal origins (Fig. 4a). Furthermore, the peaks detected in H3K27ac and H3K4me3 ChIP-seq in neuronal cells were well overlapped with open-chromatin regions defied by the publicly available ATAC-seq in neuronal cells ${ }^{18}$, suggesting the peaks of neuron-specific ChIP-seq were indeed associated with transcriptionally active regions (Fig. $4 b, c$ ). 
(a)

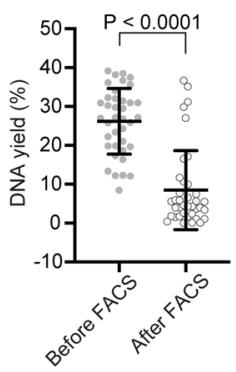

(b)

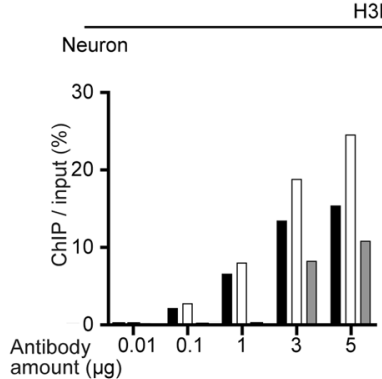

$\mathrm{H} 3 \mathrm{~K} 4 \mathrm{me} 3$
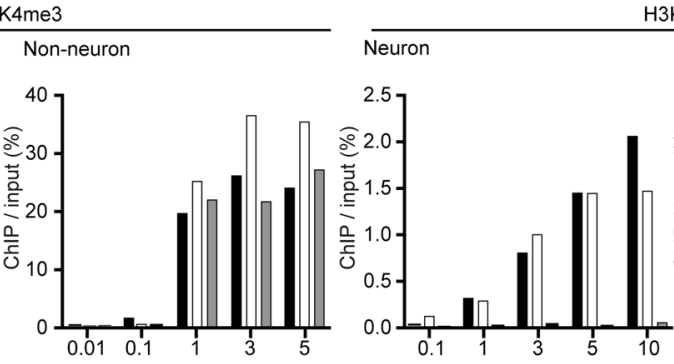

H3K27ac

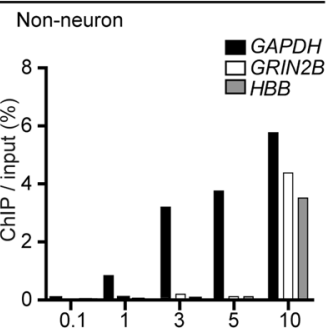

(c)

H3K4me3
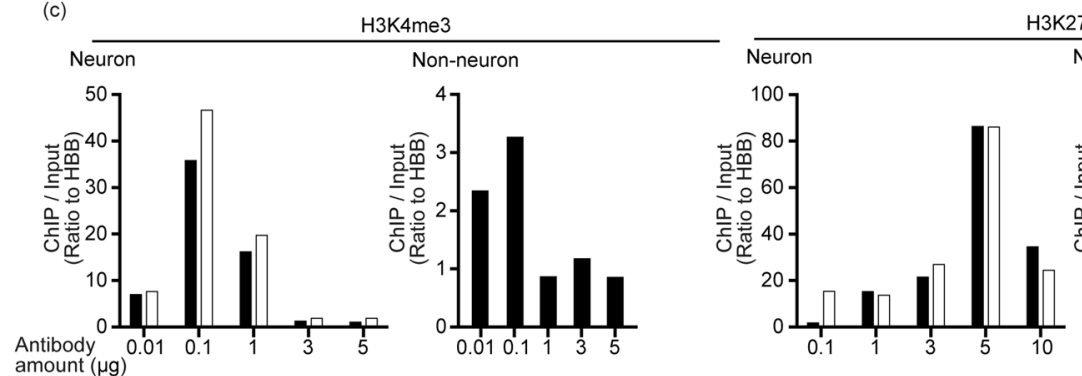

H3K27ac

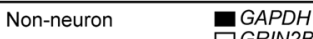

Figure 1. Optimization of fixation and immunoprecipitation. (a) The relationship between the DNA yield and the duration of crosslinking. The DNA yield was calculated based on the assumption that the amount of genomic DNA in a single human cell was $6.6 \mathrm{pg}^{35}$. Dark gray dots represent data before FACS, the light gray dots represent data after FACS, and the black bar represent mean $\pm S D$. The statistical significance was determined by $t$-test. $\mathrm{N}=38$. $(\mathbf{b}, \mathbf{c})$ The relationship between the antibody amount and fold enrichment of the house-keeping gene $(G A P D H)$, a neuronally expressed gene (GRIN2B) and non-neuronally expressed gene $(H B B)$. Fold enrichment was calculated using qPCR of enriched DNA fragment as ChIP/Input (\%) (b) or its ratio to $H B B(\mathbf{c})$.

Genome-wide profiles of neuronal and non-neuronal ChIP-seq. Next, we analyzed the genome-wide profiles of the histone modifications. Compared to the background profile of the human whole genome, H3K4me3 modifications in both the neuronal and non-neuronal samples were enriched in the active promoter regions ( $2.4 \%$ vs $24.5 \%$ and $24.8 \%$, respectively), and $\mathrm{H} 3 \mathrm{~K} 27 \mathrm{ac}$ was also enriched around TSS region, suggesting successful enrichment of each histone mark feature (Fig. 5a) ${ }^{19}$. Gene ontology analysis also supported the enrichment of neuronal ontology in $\mathrm{H} 3 \mathrm{~K} 4 \mathrm{me} 3$ and H3K27ac ChIP-seq of neuronal samples. On the other hand, non-neuronal samples showed less or no significant enrichment in H3K27ac or H3K4me3, respectively (Fig. 5b). In the clustering and principal component analysis, neuronal, non-neuronal and bulk samples were well clustered within each cell type, but the neuronal group formed a distinctive cluster from other 2 groups. The genome-wide distribution of histone modifications obtained from the bulk samples was similar to that obtained from the non-neuronal samples, but was distinctive from the neuronal samples (Fig. 5c,d), which can be attributed to the fact that the ratio of the amount of neurons to non-neurons, even in the cortex, was approximately 2:3 (Fig. S1a). This suggests that the isolation of neuronal nuclei is essential when analyzing neuronal histone modifications, and is not possible using bulk brain tissue analysis. These data support our hypothesis that ChIP-seq of bulk samples does not necessarily reflect neuronal histone modification, but rather reflects that of the non-neuronal cells. Finally, we also confirmed high reproducibility between technical replicates (the Pearson's coefficient 0.99 ) (Fig. S4), supporting the robustness of our method described above.

\section{Discussion}

Here, we established a method for neuron-specific ChIP-seq analysis. Optimization of the fixation process enhanced the yield and reproducibility of DNA extraction, and optimal amount of antibody in immunoprecipitation step increased the ChIP efficiency and minimized non-specific binding. The genome-wide profiles of histone modifications in neuronal cells have distinctive patterns compared to that of the bulk brain or non-neuronal cells, 
(a)

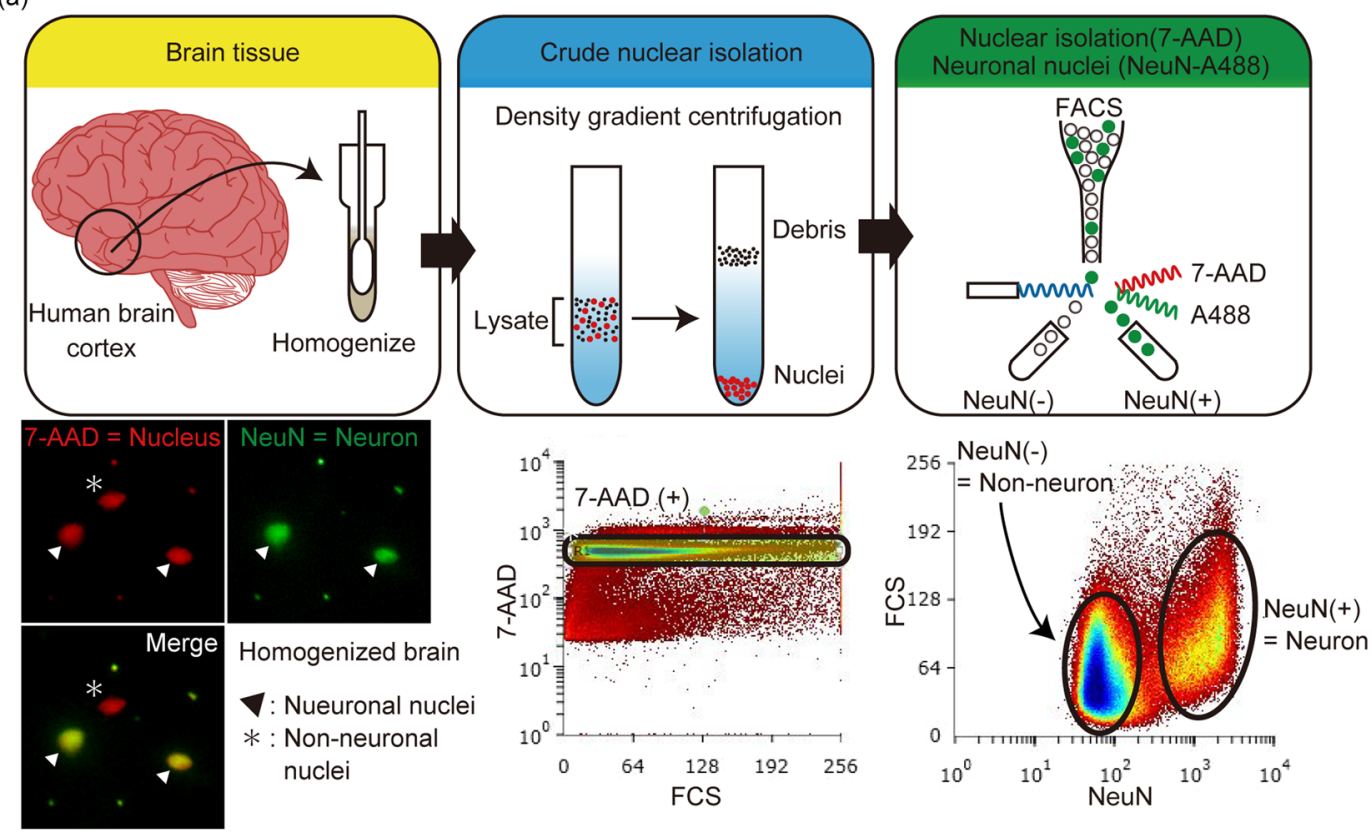

(b)
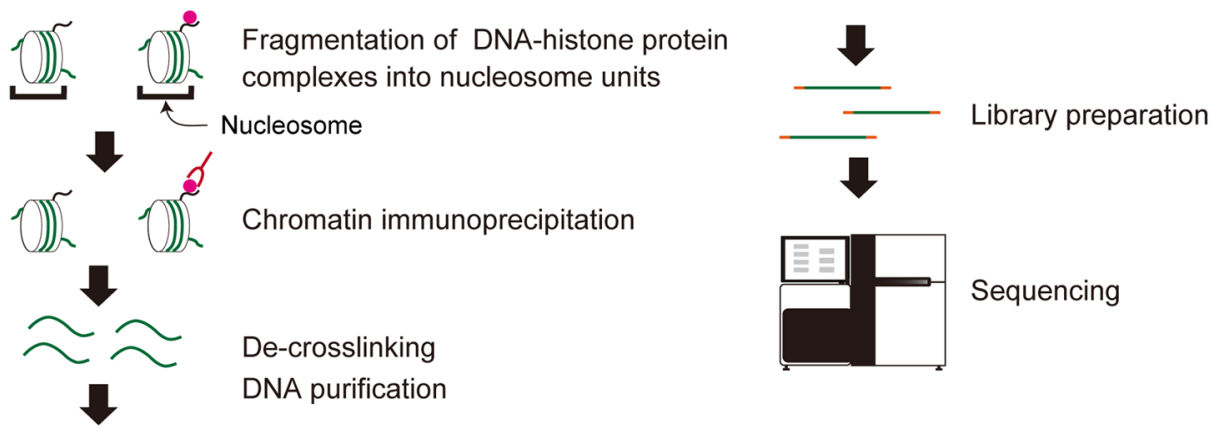

Figure 2. Schematic illustration of neuronal nuclei isolation and chromatin immunoprecipitation. (a) Isolation of neuronal nuclei using FACS. Upper panel shows the schematic process of each step, and lower panels show representative data. Brain samples were homogenized and subjected to density gradient centrifugation to obtain crude nuclear isolates. All the nuclei were stained with 7-AAD, and separation of neuronal and non-neuronal nuclei was performed by Alexa488-conjugated anti-NeuN antibody. FACS separation was performed. The lower panels are immunofluorescence images of neuronal and non-neuronal nuclei and neuronal/non-neuronal nuclei isolation using FACS. (b) Schematic diagram of ChIP. The obtained nuclei were sonicated to fragment the genomic DNA into nucleosome units. The nucleosomes with the target histone modifications were captured using an antibody against the target modification. The captured DNA was purified and subjected to library preparation and next generation sequencing.

suggesting that the genome-wide profiles of histone modification in neuronal cells cannot be estimated using that of the bulk brain.

Unlike in the DNA methylome analysis, where the covalent binding of the methyl group to cytosine retains the modification during the assay, ChIP-seq requires fixation to maintain the interaction between the DNA and the histone proteins because the DNA only wraps around the histone proteins without any covalent binding. As discussed earlier, fixation immediately following homogenization improves the yield of genomic DNA compared to fixation after FACS. This may be because the FACS process impairs the nuclear membrane, thus allowing genomic DNA to leak from the nucleus even with nuclear stabilization with calcium and magnesium supplementation.

The histone modification profile of the neuronal cells was distinctive from those of the bulk brain and non-neuronal cells. This result was consistent with DNA methylation study, where DNA methylation in the bulk brain was similar to non-neuronal cells, not to neuronal cells ${ }^{20}$. This can be attributed to the fact that the majority of cells comprising the brain are glial cells that have distinctive epigenomic feature compared to neuronal cells ${ }^{21}$. ChIP-seq. ${ }^{9}$ studies using bulk brains for Alzheimer's disease have shown that some of the differentially modified regions are associated with risk loci identified by genome-wide association study (GWAS). GWAS analyzes genomic 
(a)

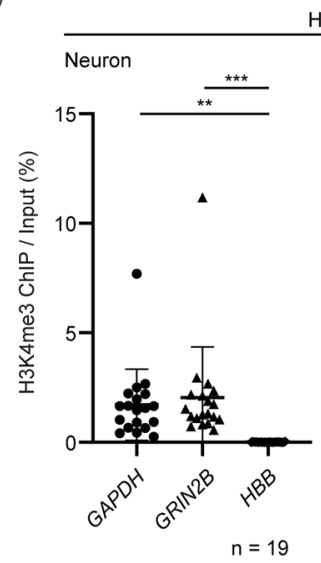

H3K4me3

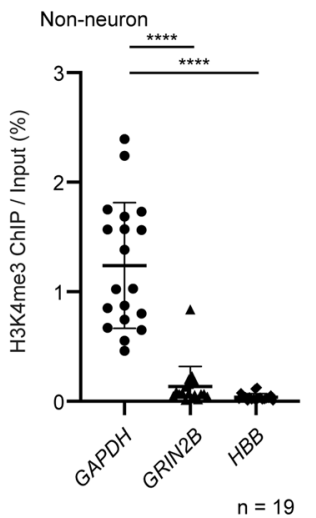

(b)

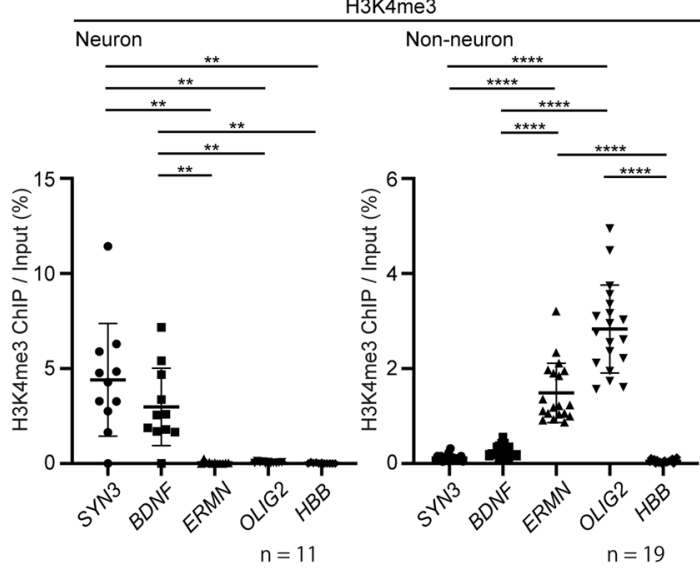

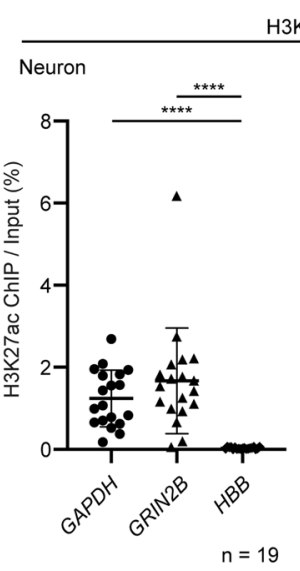

H3K27ac

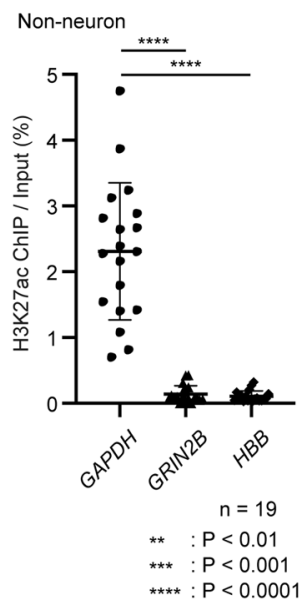

$* * *: P<0.001$

$\star \star \star \star: P<0.0001$

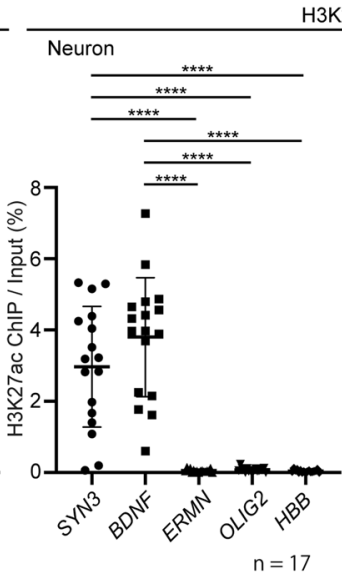

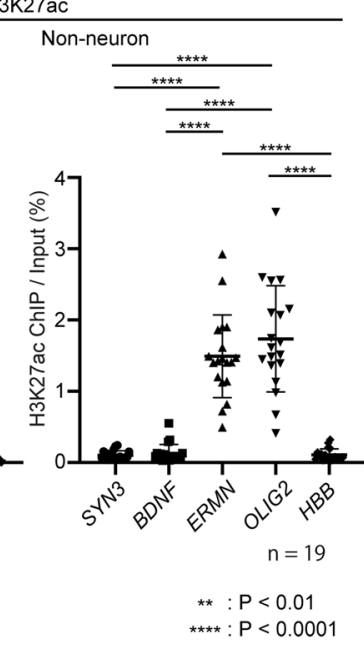

Figure 3. quantitative PCR of neuronal and non-neuronal ChIP samples. (a) To validate neuronal and nonneuronal specificity of ChIP samples, samples were subjected to qPCR for GAPDH, GRIN2B and HBB which were also used in the optimization step (Fig. 1). (b) qPCR was also performed for SYN3 and BDNF, neuronal regions, and ERMN and OLIG2, non-neuronal regions. Fold enrichment was calculated using qPCR of enriched DNA fragment as ChIP/Input (\%). The black bar represents mean \pm SD. Statistical significance was determined by one-way ANOVA with post-hoc Turkey (H3K4me3 ChIP using neurons) and Brown-Forsythe ANOVA with post-hoc Dunnett's correction (the others). The number of samples is shown in each panel. $* * \mathrm{P}<0.01$, $* * * \mathrm{P}<0.001, * * * \mathrm{P}<0.0001$.

sequences that are identical throughout the body and has no specificity to its derived cell type. Therefore, these studies suggest the possibility that epigenetic changes in the neuronal cells were obscured in the bulk analysis and epigenetic analysis using bulk brain can only extract the information that cell-type independent GWAS can identify. On the other hand, neuron-specific DNA methylation analysis of post-mortem brains from Alzheimer's disease patients could help to identify a novel pathomechanism occurring exclusively in neuronal cells ${ }^{15}$. Chromatin accessibility analysis with the Assay for Transposase Accessible Chromatin followed by sequencing (ATAC-seq) in neuronal cells from schizophrenia also provided a novel single nucleotide polymorphism (SNP) with biological relevance ${ }^{22}$. These reports suggest the importance of neuron-specific epigenetic analysis. Neuron-specific ChIP-seq can potentially shed light on novel neuronal phenomena and elucidate molecular events occurring in neuronal cells.

As for genomic annotations of ChIP-seq enrichment, the promoter regions were enriched in H3K4me3 (Fig. 4a), which was consistent with previous reports that H3K4me3 was enriched in active promoters. Notably, compared to H3K27ac, the $5^{\prime}$ UTR was also enriched in H3K4me3 ChIP-seq (in neuronal cells, $10 \%$ vs $2.6 \%$ for $\mathrm{H} 3 \mathrm{~K} 4 \mathrm{me} 3$ and $\mathrm{H} 3 \mathrm{~K} 27 \mathrm{ac}$, respectively). A recent study has shown that METTL3 is recruited to TSS characterized by H3K4me3 modifications ${ }^{23}$. METTL3 forms an N6-methyltransferase complex that co-transcriptionally deposits N6-methyladenosine $\left(\mathrm{m}^{6} \mathrm{~A}\right)$ to RNA and regulates various biological processes ${ }^{24,25}$. In neuronal cells, approximately half of expressed mRNAs are $\mathrm{m}^{6} \mathrm{~A}$ modified, and decreased $\mathrm{m}^{6} \mathrm{~A}$ impairs neurogenesis and neuronal functions, supporting the importance of $\mathrm{m}^{6} \mathrm{~A}$ deposition by METTL3 ${ }^{26}$. Our data imply that the genomic structure of $5^{\prime} \mathrm{UTR}$ regulated by $\mathrm{H} 3 \mathrm{~K} 4 \mathrm{me} 3$ might be associated with neuronal functions through METTL3 recruitment. 
(a)

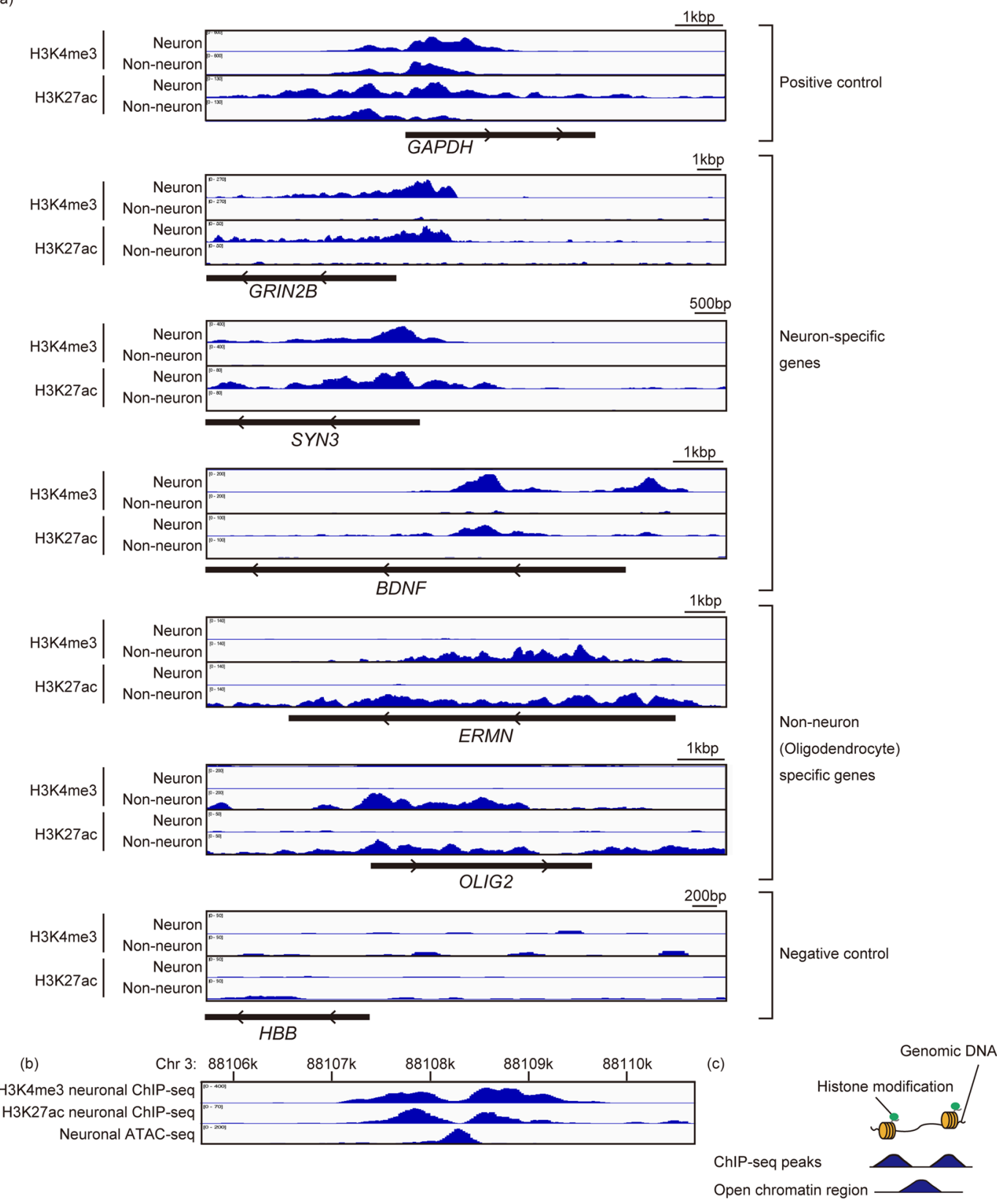

Figure 4. The distribution of mapped reads. (a) The distribution of mapped reads around TSS of GAPDH, GRIN2B and HBB. ChIP-seq peak of H3K4me3 and H3K27ac in neuronal and non-neuronal cells is displayed on the IGV browser. Black bars under the distribution of the mapped reads show the position and orientation of genes. (b) Representative distribution of ChIP-seq peaks and ATAC-seq peak ${ }^{18}$. The peaks of H3K4me 3 ChIP-seq, H3K27ac ChIP-seq were consistent with ATAC-seq peak. (c) Scheme illustrating the relationship with ChIP-seq peaks and chromatin structure. ChIP-seq peaks represent the genomic region wrapping around the histones with specific modifications, and ATAC-seq peaks represent the genomic region free from protein bindings, thus, ATAC-seq peak generally exist between the ChIP-seq peaks.

Neuronal cells extend projections called neurites to connect with each other at synapsis to form functional networks, which is a distinctive feature unlike in other systemic organs where their functions are dependent on the cell number. Neuronal network is the structural basis for memory and learning process, and previous reports have demonstrated that histone modifications play a pivotal role ${ }^{27-31}$. Consistent with these reports, the two histone modifications analyzed in this study in neuronal cells showed prominent enrichment of the ontology terms associated with synapse and neurite projection. Non-neuronal cells also showed enrichment of the terms associated with the environment of the central nervous system maintaining neuronal and glial functions. However, their enrichment was much weaker than that of neuronal cells, which may be attributed to the fact that non-neuronal cells are a heterogeneous cell population and includes astrocytes, oligodendrocytes, and vascular cells. 
(a)
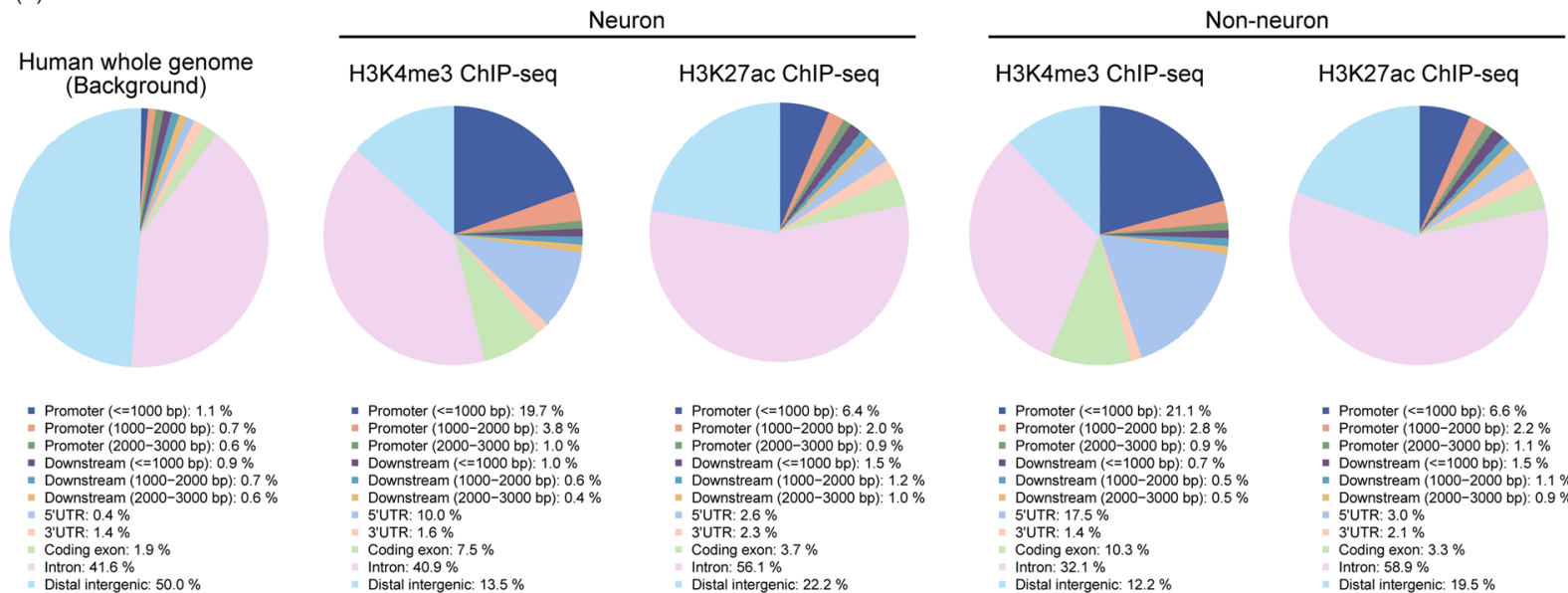

Promoter $(<=1000 \mathrm{bp}): 19.7 \%$

E Promoter (1000-2000 bp): $3.8 \%$

II Promoter (2000-3000 bp): $1.0 \%$

"Promoter $(2000-3000 \mathrm{bp}): 0.9 \%$

- Downstream (<=1000 bp): $1.5 \%$

$\begin{array}{ll}\text { Downstream (1000-2000 bp): } 0.6 \% & \text { "Downstream (1000-2000 bp): } 1.2 \% \\ \text { Downstream (2000-3000 bp): } 0.4 \% & \text { "Downstream (2000-3000 bp): } 10 \%\end{array}$

5'UTR: $10.0 \%$

3'UTR: $1.6 \%$
Coding exon: $7.5 \%$

Distal intergenic: $13.5 \%$

" 5'UTR: $2.6 \%$

3'UTR: $2.3 \%$

Coding exon: $3.7 \%$
Intron: $56.1 \%$

Intron: $56.1 \%$
Distal intergenic: $22.2 \%$

- Promoter (c=1000 bp): $21.1 \%$

Promoter (1000-2000 bp): $2.8 \%$

- Promoter (2000-3000 bp): $0.9 \%$

- Downstream (1000-2000 bp): $0.5 \%$

" Downstream (2000-

3'UTR: $1.4 \%$

Coding exon: $10.3 \%$
Intron: $32.1 \%$

Distal intergenic: $12.2 \%$

- Promoter (1000-2000 bp): $2.2 \%$

- Downstream (<=1000 bp) $15 \%$

- Downstream (1000-2000 bp): $1.1 \%$

Downstream (2000-3000 bp): $0.9 \%$

5'UTR: $3.0 \%$

Coding exon: $3.3 \%$

Coding exon: $3.3 \%$
Intron: $58.9 \%$

Distal intergenic: $19.5 \%$

(b)

Neuron

H3K4me3

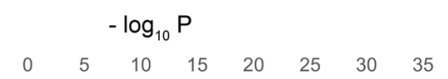

H3K27ac

$-\log _{10} P$

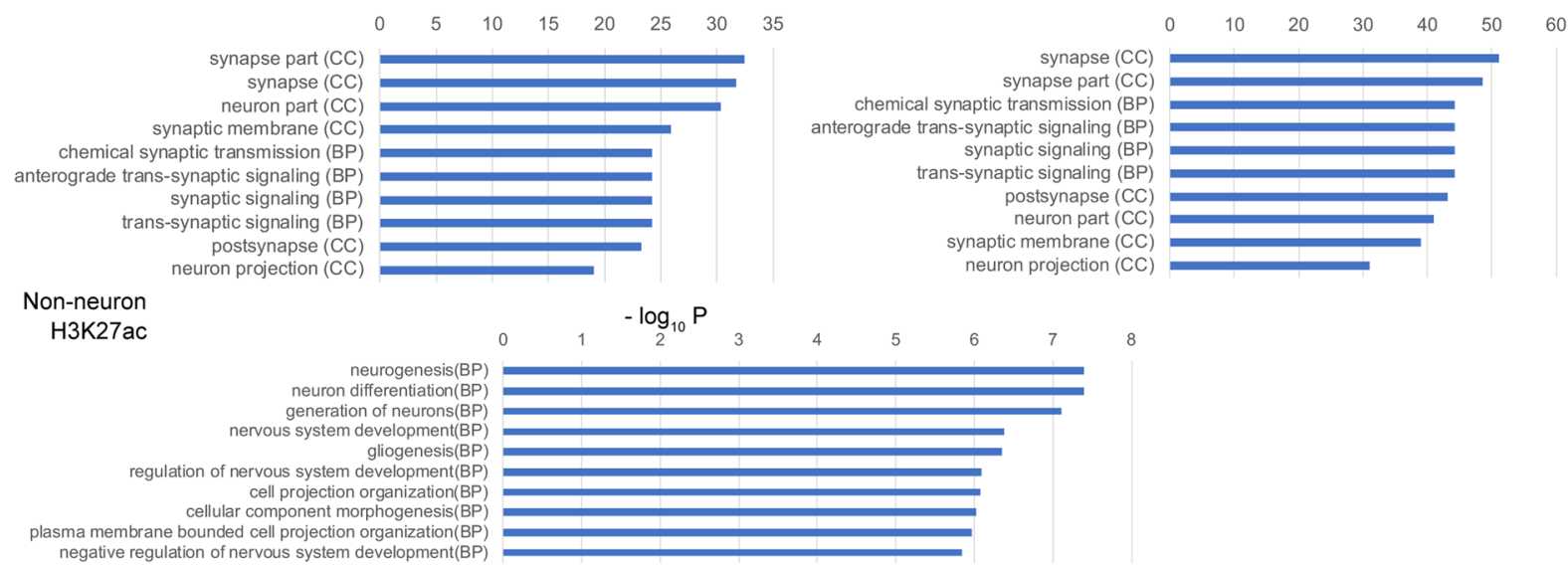

(c)

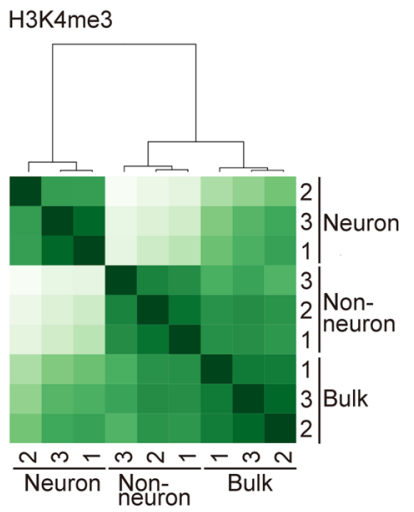

(d)

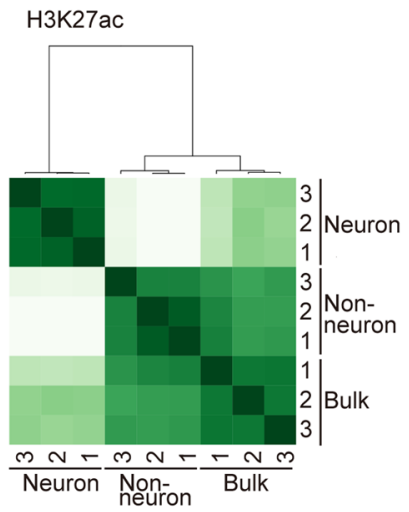

H3K4me3

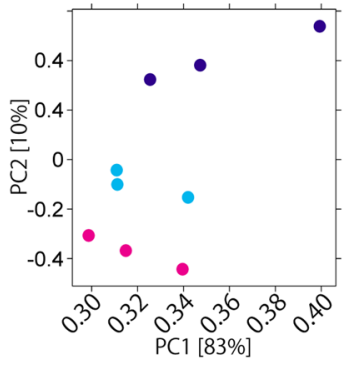

H3K27ac

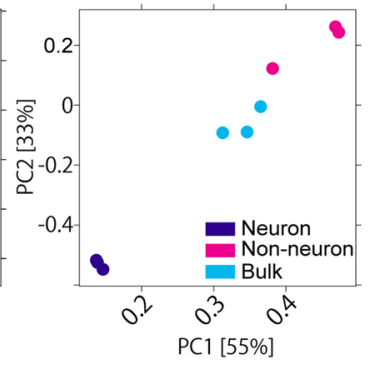

Figure 5. The genome-wide profiles of $\mathrm{H} 3 \mathrm{~K} 4 \mathrm{me} 3$ and H3K27ac ChIP-seq peaks in neuronal cells, nonneuronal cells and bulk brain. (a) Genomic annotation of $\mathrm{H} 3 \mathrm{~K} 4 \mathrm{me} 3$ and $\mathrm{H} 3 \mathrm{~K} 27 \mathrm{ac}$ profiles in neuronal and non-neuronal cells. Each ChIP-seq peak was analyzed by CEAS. (b) The top 10 significantly enriched GO terms (y-axis) and their $\mathrm{p}$-values ( $\mathrm{x}$-axis) of differentially modified genes. Only the terms with statistical significance $(\mathrm{P}<0.01)$ are shown, such that no terms are shown for H3K4me3 ChIP-seq of non-neuronal samples. (c) Clustering analysis of ChIP-seq peak in neuronal, non-neuronal cells and bulk brain. (d) Principal component analysis of ChIP-seq peak in neuronal, non-neuronal cells and bulk brain.

Neuron-specific ChIP-seq is advantageous in that it allows us to summarize the genomic profile of histone modifications in many neuronal cells and extract common and representative changes among them. However, this assay abolishes the heterogeneity of neuronal cells in the summarization process, which in some cases could 
be a disadvantage. For example, only a part of the excitatory neurons is activated in physiological conditions. Neuronal cells can be categorized into several subpopulations and some intact cells remain even in the advanced stages of Alzheimer's disease. The recently developed single-cell ChIP-seq technique overcomes such heterogeneity issues to successfully identify even sub-populations within a supposedly single-type of cell population ${ }^{32}$. However, technical limitations such as insufficient coverage narrows the detectable range to the genes with higher expression, and still prevent discrimination of phenotype-specific alterations from variations within cell types. Cell-type specific ChIP-seq collects histone modifications data from each cell to converge the data and minimize variations within cell types, and thus can predict the transcriptional changes in genes with low expression that is more suitable for the study of neurodegenerative disorders.

In summary, we established a method for neuron-specific ChIP-seq using post-mortem brain samples. The optimization of the fixation and immunoprecipitation conditions enables ChIP-seq to be highly specific to neuronal cells, with enhanced enrichment and reproducibility. Neuron-specific ChIP-seq will expand our understanding of neuronal plasticity and the neurodegenerative process.

\section{Materials and Methods}

Human brain samples. This research was approved by the ethics committee of the University of Tokyo (approval \#2183-17). All human samples were used in accordance with the principles of the Declaration of Helsinki.

We collected postmortem brains with written consent from the patients' families and maintained them at $-80^{\circ} \mathrm{C}$ until use. The brain samples from 22 normal subjects were obtained from The University of Tokyo, Tokyo Metropolitan Geriatric Hospital brain bank and Tsukuba University, of which 3 samples were subjected to ChIP-seq and 19 samples were subjected to ChIP with qPCR analysis. Trained neuropathologists made a pathological diagnosis of the brains and confirmed no pathological changes in the brain. The detailed demographics of the brain samples are shown in Supplementary Tables 1 and 2.

Neuronal/Non-neuronal nuclei isolations. Human brain cortex were dissected from the temporal or parietal lobes (500 mg - 1,000 mg per assay), and homogenized using a Teflon homogenizer (AS ONE, Osaka, Japan) in nuclear buffer $(10 \mathrm{mM}$ Tris- $\mathrm{HCl} \mathrm{pH} 8.0,0.32 \mathrm{M}$ sucrose, $5 \mathrm{mM} \mathrm{CaCl}, 3 \mathrm{mM} \mathrm{MgAc} 2,0.1 \mathrm{mM}$ EDTA and $0.1 \%(\mathrm{v} / \mathrm{v})$ Triton X-100) supplemented with a protease inhibitor cocktail (Roche, Basel, Switzerland). Brain homogenates were subjected to a Percoll (GE Healthcare, Pittsburgh, PA, USA) step gradient on four-layers (12\%, $17.4 \%, 26 \%$, and $35 \%$ ), centrifuged at $16,000 \times g$ for $30 \mathrm{~min}$ at $4{ }^{\circ} \mathrm{C}$, and the nuclei were collected from the $35 \%$ Percoll fraction. The isolated nuclei were blocked with $1.5 \%$ bovine serum albumin (BSA) and stained with Alexa Flour 488-conjugated anti-NeuN antibody (Millipore, Darmstadt, Germany, 1:500, clone A60, AB_2149209) and $15 \mu \mathrm{g} / \mathrm{mL}$ of 7-amino-actinomycin D (7-AAD, BioLegend, San Diego, USA) overnight at $4{ }^{\circ} \mathrm{C}$, and subjected to fluorescence-activated cell sorting (FACS). The nuclei were first gated on the basis of 7-AAD intensity, where all the nuclei were stained equally, and then subjected to separation of the neuronal and non-neuronal nuclei by NeuN-A488 intensity (Fig. 2). To stabilize the sorted nuclei, $\mathrm{MgAc}_{2}$ and $\mathrm{CaCl}_{2}$ solutions were added to post-FACS nuclei samples (final concentrations: $0.3 \mathrm{M}$ sucrose, $\left.2.4 \mathrm{mM} \mathrm{MgAce}, 24 \mathrm{mM} \mathrm{CaCl}_{2}\right)^{33}$. To crosslink the DNA and histone proteins, the nuclei were fixed immediately after homogenization or nuclei sorting depending on the methods.

Western blotting. Isolated nuclei were resuspended with $1 \times$ lithium dodecyl sulfate (LDS) buffer (Invitrogen, Carlsbad, CA, USA) and $1 \%$ of 2-Mercaptoethanol and incubated at $60^{\circ} \mathrm{C}$ with for $15 \mathrm{~min}$. Samples were resolved on SDS-PAGE, and the separated proteins were transferred to polyvinylidene difluoride (PVDF) membranes using a Trans-Blot Turbo Blotting System and Trans-Blot Turbo Transfer Pack (Bio-Rad, Hercules, CA, USA). Non-specific binding was blocked with EzBlock CAS (ATTO Corporation, Tokyo, Japan) for 60 min at $25^{\circ} \mathrm{C}$. Membranes were then incubated with primary antibodies in CanGetSignal Solution I (TOYOBO, Osaka, Japan) for $2 \mathrm{~h}$ at $25^{\circ} \mathrm{C}$ or overnight at $4{ }^{\circ} \mathrm{C}$. Anti-NeuN (A60, Millipore Cat\# A60, RRID:AB_2314889, 1:1000) and anti-Olig2 (Millipore Cat\# AB9610, RRID:AB_570666, 1:1000) were used as primary antibodies. Membranes were washed with TBST for $10 \mathrm{~min}$ three times, and then incubated with secondary horseradish peroxidase-conjugated antibodies (GE Healthcare, Little Chalfont, UK), diluted 1:2000 in TBST for $1 \mathrm{~h}$ at $25^{\circ} \mathrm{C}$. After membranes were washed with Tris-buffered saline (TBS-T) for $10 \mathrm{~min}$ three times, they were visualized by EzWestLumi Plus (ATTO). Images were captured by LuminoGraph I (ATTO), and quantified using Image J software (http://imagej.nih.gov/ij/).

Fragmentation of DNA-histone protein complexes into nucleosome units. The isolated nuclei were centrifuged at $4,000 \mathrm{rpm} 4^{\circ} \mathrm{C}$ for $5 \mathrm{~min}$, resuspended in pre-chilled lysis buffer $1(50 \mathrm{mM} \mathrm{HEPES}-\mathrm{KOH} \mathrm{pH}$ $7.5,140 \mathrm{mM} \mathrm{NaCl}, 1 \mathrm{mM}$ EDTA pH 8.0, $10 \% \mathrm{w} / \mathrm{v}$ glycerol, $0.5 \% \mathrm{w} / \mathrm{v}$ NP-40, 0.25\% w/v Triton X-100), and incubated at $4{ }^{\circ} \mathrm{C}$ for $10 \mathrm{~min}$. Subsequently, the nuclei were resuspended in pre-chilled lysis buffer $2(10 \mathrm{mM} \mathrm{Tris}-\mathrm{HCl}$ pH 8.0, $200 \mathrm{mM} \mathrm{NaCl}, 1 \mathrm{mM}$ EDTA pH 8.0, $0.5 \mathrm{mM}$ EGTA pH 8.0) and lysis buffer 3 (50 mM Tris- $\mathrm{HCl} \mathrm{pH}$ 8.0, $1 \%$ SDS, 10 mM EDTA pH 8.0), sequentially, according to a previously published protocol ${ }^{34}$. The nuclei were sonicated using a Focused-ultrasonicator (Covaris E220 (Covaris, Woburn, MA, USA), duty: 5.0\%, Intensity/ peak incident power (PIP): 105, Cycles per burst: 200, Treatment time: 1200 seconds) with its genomic DNA becoming 150-250 bp in length (Fig. S1). The fragmented DNA solution was centrifuged at $16,000 \times g$ for 5 min at $4^{\circ} \mathrm{C}$ to remove the debris, and the supernatant was aliquoted into the corresponding amounts $\left(2 \times 10^{6}\right.$ nuclei per immunoprecipitation and $2 \times 10^{5}$ nuclei per input sample). The fragmented DNA samples were frozen at $-80^{\circ} \mathrm{C}$ until further processing. 
Chromatin immunoprecipitation and DNA purification. Prior to immunoprecipitation, the lysates corresponding to $2 \times 10^{6}$ nuclei were diluted to $1 \mathrm{~mL}$ volume with the ChIP dilution buffer $(16.7 \mathrm{mM}$ Tris- $\mathrm{HCl}$ pH $8.0,0.01 \%$ SDS, $1.1 \%$ Triton X-100, $1.2 \mathrm{mM}$ EDTA pH 8.0, $167 \mathrm{mM} \mathrm{NaCl}$ ). Anti-histone antibodies (anti-H3K4me3 antibody, abcam, \#8580; anti-H3K27ac antibody, Active motif, \#39133) were added to the samples, and incubated at $4{ }^{\circ} \mathrm{C}$ for 4 hours. Then, $40 \mu \mathrm{g}$ of pre-washed magnetic beads (Dynabeads Protein $\mathrm{G}$ for H3K27ac or Dynabeads Protein A (Life Technologies) for H3K4me3) were added to capture antibody-histone complexes and incubated at $4^{\circ} \mathrm{C}$ for 1 hour. The beads were then washed with $1 \mathrm{~mL}$ of pre-chilled Low salt buffer (20 mM Tris- $\mathrm{HCl} \mathrm{pH} \mathrm{8.0,} \mathrm{0.1 \%} \mathrm{SDS,} 1 \% \mathrm{w} / \mathrm{v}$ Triton X-100, 2 mM EDTA pH 8.0, $150 \mathrm{mM} \mathrm{NaCl}$ ) 5 times followed by washing in $1 \mathrm{~mL}$ of pre-chilled High salt buffer ( $20 \mathrm{mM}$ Tris- $\mathrm{HCl} \mathrm{pH} 8.0,0.1 \% \mathrm{SDS}, 1 \% \mathrm{w} / \mathrm{v}$ Triton X-100, $2 \mathrm{mM}$ EDTA $\mathrm{pH} 8.0,500 \mathrm{mM} \mathrm{NaCl}) 3$ times. The immunoprecipitated nucleosome complexes were eluted with $200 \mu \mathrm{L}$ of ChIP Elution Buffer (10 mM Tris- $\mathrm{HCl} \mathrm{pH} \mathrm{8.0,300} \mathrm{mM} \mathrm{NaCl,} 5 \mathrm{mM}$ EDTA pH 8.0, 1\% SDS) for $15 \mathrm{~min}$ at room temperature, and eluted samples were incubated at $65^{\circ} \mathrm{C}$ overnight for de-crosslinking. The extracted DNA fragments were purified using a standard phenol/chloroform/isoamyl alcohol protocol.

Quantitative PCR analysis. Quantitative real-time polymerase chain reactions (RT-qPCR) were performed to assess the enrichment of neuron-specific and histone modification-specific genome regions. GPCR primers were designed for the genome regions around the transcription start site (TSS) of GAPDH as housekeeping gene, $H B B$ as a gene with low expression in the brain, GRIN2B, SYN3 and BDNF as genes with high expression only in neuronal cells, and ERMN and OLIG2 as genes with high expression only in non-neuronal cells (Table S3). qPCR was performed using PowerUp SYBR Green Master Mix (Thermo Fisher Scientific, CA, USA) with the Applied Biosystems 7900 Fast Time PCR system. The enrichments were calculated using the percent input method that is the signals obtained from the ChIP samples are divided by the signals obtained from the input samples.

Library preparation and NGS sequencing. Input and immunoprecipitated DNA samples were subjected to end repair, A-tailing, adapter ligation, and amplification using KAPA Hyper Prep kit (KAPA Biosystems, Cape Town, South Africa) according to the manufacturer's instructions. The library thus obtained was cleaned up with Agencourt AMPure XP (Beckman Coulter, Fullerton, CA, USA) and quantified using qPCR with KAPA Library Quantification kit (KAPA Biosystems) prior to sequencing. The libraries for technical replicates were sequenced on MiSeq (Illumina, San Diego, CA, USA) to obtain 20 million paired end reads (75 base pair), and libraries for neuron- and non-neuron specific ChIP-seq were sequenced on Hi-Seq2500 (Illumina) to obtain 40 million paired end reads (100 base pair).

Data processing. The initial quality control and adaptor trimming were performed using Trimmomatic v.0.36 with standard parameters. The reads were mapped to the reference human genome (hg19) using Bowtie2 v.2.3.4 and subjected to peak calling using MACS2 v.2.1.1 with a q-value threshold of 0.01. The distribution of the mapped reads over the genome features was analyzed using CEAS v.1.0.2 for neurons. The differential binding and gene ontology analysis were performed using R 3.4.3/Bioconductor v.3.6 packages DiffBind v.2.6.6 and ChIPPeakAnno v.3.6.5, respectively. The reproducibility of the replicates of neuron-specific ChIP-seq was assessed using deeptools v.3.2.1. ATAC-seq (Assay for Transposase-Accessible Chromatin using sequencing) using the pre-frontal cortex was analyzed with publicly available data ${ }^{18}$.

Received: 18 September 2019; Accepted: 17 February 2020;

Published online: 28 February 2020

\section{References}

1. Cedar, H., Bergman, Y. \& Linking, D. N. A. methylation and histone modification: patterns and paradigms. Nat. Rev. Genet. 10, 295-304, https://doi.org/10.1038/nrg2540 (2009).

2. Kovacheva, V. P. et al. Gestational choline deficiency causes global and Igf2 gene DNA hypermethylation by up-regulation of Dnmt1 expression. J. Biol. Chem. 282, 31777-31788, https://doi.org/10.1074/jbc.M705539200 (2007).

3. Weaver, I. C. et al. The transcription factor nerve growth factor-inducible protein a mediates epigenetic programming: altering epigenetic marks by immediate-early genes. J. Neurosci. 27, 1756-1768, https://doi.org/10.1523/JNEUROSCI.4164-06.2007 (2007).

4. Fischer, A., Sananbenesi, F., Wang, X., Dobbin, M. \& Tsai, L. H. Recovery of learning and memory is associated with chromatin remodelling. Nature 447, 178-182, https://doi.org/10.1038/nature05772 (2007).

5. Williams, B. M. et al. Environmental enrichment: effects on spatial memory and hippocampal CREB immunoreactivity. Physiol. Behav. 73, 649-658, https://doi.org/10.1016/s0031-9384(01)00543-1 (2001).

6. Benayoun, B. A. et al. Remodeling of epigenome and transcriptome landscapes with aging in mice reveals widespread induction of inflammatory responses. Genome Res. 29, 697-709, https://doi.org/10.1101/gr.240093.118 (2019).

7. Sen, P., Shah, P. P., Nativio, R. \& Berger, S. L. Epigenetic Mechanisms of Longevity and Aging. Cell 166, 822-839, https://doi. org/10.1016/j.cell.2016.07.050 (2016).

8. Pu, M., Wang, M., Wang, W., Velayudhan, S. S. \& Lee, S. S. Unique patterns of trimethylation of histone H3 lysine 4 are prone to changes during aging in Caenorhabditis elegans somatic cells. PLoS Genet. 14, e1007466, https://doi.org/10.1371/journal. pgen.1007466 (2018)

9. Marzi, S. J. et al. A histone acetylome-wide association study of Alzheimer's disease identifies disease-associated H3K27ac differences in the entorhinal cortex. Nat. Neurosci. 21, 1618-1627, https://doi.org/10.1038/s41593-018-0253-7 (2018).

10. Klein, H. U. et al. Epigenome-wide study uncovers large-scale changes in histone acetylation driven by tau pathology in aging and Alzheimer's human brains. Nat. Neurosci. 22, 37-46, https://doi.org/10.1038/s41593-018-0291-1 (2019).

11. Azevedo, F. A. et al. Equal numbers of neuronal and nonneuronal cells make the human brain an isometrically scaled-up primate brain. J. Comp. Neurol. 513, 532-541, https://doi.org/10.1002/cne.21974 (2009).

12. von Bartheld, C. S., Bahney, J. \& Herculano-Houzel, S. The search for true numbers of neurons and glial cells in the human brain: A review of 150 years of cell counting. J. Comp. Neurol. 524, 3865-3895, https://doi.org/10.1002/cne.24040 (2016). 
13. Andrade-Moraes, C. H. et al. Cell number changes in Alzheimer's disease relate to dementia, not to plaques and tangles. Brain 136, 3738-3752, https://doi.org/10.1093/brain/awt273 (2013).

14. Park, P. J. ChIP-seq: advantages and challenges of a maturing technology. Nat. Rev. Genet. 10, 669-680, https://doi.org/10.1038/ nrg2641 (2009).

15. Mano, T. et al. Neuron-specific methylome analysis reveals epigenetic regulation and tau-related dysfunction of BRCA1 in Alzheimer's disease. Proc. Natl Acad. Sci. USA 114, E9645-E9654, https://doi.org/10.1073/pnas.1707151114 (2017).

16. He, H. H. et al. Nucleosome dynamics define transcriptional enhancers. Nat. Genet. 42, 343-347, https://doi.org/10.1038/ng.545 (2010).

17. Jiang, Y., Matevossian, A., Huang, H. S., Straubhaar, J. \& Akbarian, S. Isolation of neuronal chromatin from brain tissue. BMC Neurosci. 9, 42, https://doi.org/10.1186/1471-2202-9-42 (2008).

18. Fullard, J. F. et al. An atlas of chromatin accessibility in the adult human brain. Genome Res. 28, 1243-1252 (2018).

19. Heintzman, N. D. et al. Distinct and predictive chromatin signatures of transcriptional promoters and enhancers in the human genome. Nat. Genet. 39, 311-318, https://doi.org/10.1038/ng1966 (2007).

20. Iwamoto, K. et al. Neurons show distinctive DNA methylation profile and higher interindividual variations compared with nonneurons. Genome Res. 21, 688-696, https://doi.org/10.1101/gr.112755.110 (2011).

21. Lunnon, K. et al. Methylomic profiling implicates cortical deregulation of ANK1 in Alzheimer's disease. Nat. Neurosci. 17, 1164-1170, https://doi.org/10.1038/nn.3782 (2014).

22. Fullard, J. F. et al. Open chromatin profiling of human postmortem brain infers functional roles for non-coding schizophrenia loci. Hum. Mol. Genet. 26, 1942-1951, https://doi.org/10.1093/hmg/ddx103 (2017).

23. Barbieri, I. et al. Promoter-bound METTL3 maintains myeloid leukaemia by m(6)A-dependent translation control. Nature 552, 126-131, https://doi.org/10.1038/nature24678 (2017).

24. Ianniello, Z., Paiardini, A. \& Fatica, A. N(6)-Methyladenosine (m(6)A): A Promising New Molecular Target in Acute Myeloid Leukemia. Front. Oncol. 9, 251, https://doi.org/10.3389/fonc.2019.00251 (2019).

25. Deng, X. et al. RNA N(6)-methyladenosine modification in cancers: current status and perspectives. Cell Res. 28, 507-517, https:// doi.org/10.1038/s41422-018-0034-6 (2018).

26. Engel, M. et al. The Role of m(6)A/m-RNA Methylation in Stress Response Regulation. Neuron 99, 389-403 e389, https://doi. org/10.1016/j.neuron.2018.07.009 (2018).

27. Swank, M. W. \& Sweatt, J. D. Increased histone acetyltransferase and lysine acetyltransferase activity and biphasic activation of the ERK/RSK cascade in insular cortex during novel taste learning. J. Neurosci. 21, 3383-3391 (2001).

28. Levenson, J. M. et al. Regulation of histone acetylation during memory formation in the hippocampus. J. Biol. Chem. 279, 40545-40559, https://doi.org/10.1074/jbc.M402229200 (2004).

29. Alarcon, J. M. et al. Chromatin acetylation, memory, and LTP are impaired in CBP $+/$ - mice: a model for the cognitive deficit in Rubinstein-Taybi syndrome and its amelioration. Neuron 42, 947-959, https://doi.org/10.1016/j.neuron.2004.05.021 (2004).

30. Korzus, E., Rosenfeld, M. G. \& Mayford, M. CBP histone acetyltransferase activity is a critical component of memory consolidation. Neuron 42, 961-972, https://doi.org/10.1016/j.neuron.2004.06.002 (2004).

31. Bourtchouladze, R. et al. A mouse model of Rubinstein-Taybi syndrome: defective long-term memory is ameliorated by inhibitors of phosphodiesterase 4. Proc. Natl Acad. Sci. USA 100, 10518-10522, https://doi.org/10.1073/pnas.1834280100 (2003).

32. Rotem, A. et al. Single-cell ChIP-seq reveals cell subpopulations defined by chromatin state. Nat. Biotechnol. 33, 1165-1172, https:// doi.org/10.1038/nbt.3383 (2015).

33. Kundakovic, M. et al. Practical Guidelines for High-Resolution Epigenomic Profiling of Nucleosomal Histones in Postmortem Human Brain Tissue. Biol. Psychiatry 81, 162-170, https://doi.org/10.1016/j.biopsych.2016.03.1048 (2017).

34. Lee, T. I., Johnstone, S. E. \& Young, R. A. Chromatin immunoprecipitation and microarray-based analysis of protein location. Nat. Protoc. 1, 729-748, https://doi.org/10.1038/nprot.2006.98 (2006).

35. Serth, J., Kuczyk, M. A., Paeslack, U., Lichtinghagen, R. \& Jonas, U. Quantitation of DNA extracted after micropreparation of cells from frozen and formalin-fixed tissue sections. Am. J. Pathol. 156, 1189-1196, https://doi.org/10.1016/S0002-9440(10)64989-9 (2000).

\section{Acknowledgements}

This study was supported by AMED under Grant Number 17dm0107069h0002, 18dm0107103 and 19dk0207046h0001, and JSPS KAKENHI Grant Number 16H05316, 16H06277 and 19K17027, The Takeda Science Foundation and The Mochida Memorial Foundation for Medical and Pharmaceutical Research.

\section{Author contributions}

K.M., T.M. and A.I. conceived and designed the experiments. K.M. and T.M. performed experiments and carried out bioinformatic analyses. M.M., S.M. and A.T. contributed materials. S.T. and T.T. supervised the study. K.M., T.M. and A.I. wrote the manuscript.

\section{Competing interests}

The authors declare no competing interests.

\section{Additional information}

Supplementary information is available for this paper at https://doi.org/10.1038/s41598-020-60775-Z.

Correspondence and requests for materials should be addressed to A.I.

Reprints and permissions information is available at www.nature.com/reprints.

Publisher's note Springer Nature remains neutral with regard to jurisdictional claims in published maps and institutional affiliations. 
(c) (i) Open Access This article is licensed under a Creative Commons Attribution 4.0 International License, which permits use, sharing, adaptation, distribution and reproduction in any medium or format, as long as you give appropriate credit to the original author(s) and the source, provide a link to the Creative Commons license, and indicate if changes were made. The images or other third party material in this article are included in the article's Creative Commons license, unless indicated otherwise in a credit line to the material. If material is not included in the article's Creative Commons license and your intended use is not permitted by statutory regulation or exceeds the permitted use, you will need to obtain permission directly from the copyright holder. To view a copy of this license, visit http://creativecommons.org/licenses/by/4.0/.

(C) The Author(s) 2020 